\title{
Biopsychosocial Risk and Protective Factors for Adolescents' Psychological Wellbeing during COVID-19: A Scoping Review
}

\section{Gamble JH*}

National Chiayi University, Taiwan

*Corresponding author: Jeffrey Hugh Gamble, National Chiayi University, 85 Wenlong Village, Minhsiung County, Chiayi, Taiwan, Tel: 886-5-226-3411 or 8216; Fax: 886-5-206-3072; Email: gamble@mail.ncyu.edu.tw

\section{Review Article}

Volume 4 Issue 6

Received Date: November 26, 2021

Published Date: December 09, 2021

DOI: $10.23880 /$ jqhe-16000250

\section{Abstract}

Background: The impact of COVID-19 on psychological well-being has gained the increasing attention of scholars and mental health practitioners. Given the fears, restrictions, and lifestyle changes resulting from the COVID-19 pandemic, adolescents are a vulnerable population in terms of psychological well-being. Moreover, given the emergence of new and more transmissible variants of SARS-CoV-2, such as the omicron variant (B.1.1.529), preparations for third, fourth, and further waves of the pandemic and accompanying upheavals to daily life must be made, including actions to protect vulnerable populations, such as adolescents.

Objectives: The purpose of this scoping review was to evaluate the protective and risk factors which influence the impact of COVID-19 on adolescents' psychological well-being in order to, in turn, provide clearer suggestions and implications for healthcare professionals.

Eligibility Criteria: Studies included in this review were required to meet the following criteria: include empirical findings related to either risk or protective factors for psychological wellbeing; be reported in the context of the COVID-19 pandemic; include adolescent participants; and be available in full-text in the English language.

Results: From a total of 6,696 articles, after excluding those with missing data, not being available in English, using nonempirical methods, or not being relevant to adolescents psychological wellbeing in the context of COVID-19, a total of 32 articles were selected for inclusion. Biological factors included gender, physical and mental disorders, and healthy habits and behaviors. Psychological factors included coping strategies, worry, resilience, other cognitive factors, and proactive behaviors. Social factors, including COVID-19 cases in the community, social support, family functioning or conflicts in the home, and routines.

Conclusion: Implications for practitioners are provided, including care in making decisions regarding opening of schools, promotion of basic health behaviors, allocating resources to individuals with pre-existing physical and mental conditions, proving educational and financial resources for parents at risk of family dysfunction, and the provision of teacher support to reduce negative emotions and develop adolescents' resilience.

Keywords: COVID-19; Psychological Well-Being; Risk Factors; Protective Factors; Adolescents; Scoping Review; Biopsychosocial Risk 


\section{Introduction}

\section{Rationale}

As a result of the COVID-19 pandemic, several measures have been taken which have directly impacted the lives of adolescents, including school closures, quarantines, lockdowns, social distancing, mask mandates, and limitations on movement $[1,2]$. As such, recent research has placed increasing emphasis on the potentially long-lasting effects of COVID-19 and related restrictions and lifestyle changes on psychological wellbeing [3]. The impact of COVID-19 on adolescent psychological wellbeing, in particular, is an increasing concern among both academics and practitioners. Given the increasing attention to the issue of psychological wellbeing, in particular, this study adopts a scoping review protocol for the evaluation of the potential risk and protective factors which are evidenced to play a role in the relationship between the pandemic and adolescents' psychological wellbeing. While several studies have already been published, focusing on specific psychological, biological/physical, or social factors, there is a lack of an updated review of the literature from a biopsychosocial perspective.

\section{Objectives}

As such, the present study intends to (a) identify protective and risk factors related specifically to adolescent psychological wellbeing during the COVID-19 pandemic, (b) evaluate the roles of biological, psychological, and social factors, and (c) provide relevant findings that can inform healthcare policy and practice, as well as to contribute to the growing literature on adolescent psychological wellbeing during COVID-19.

\section{Methods}

\section{Review Protocols}

The scoping review outlined in this report follows the JBI guidelines for scoping reviews [4]. The protocol follows the Preferred Reporting Items for Systematic Reviews and MetaAnalyses (PRISMA) checklist provided for scoping reviews [5]. The design of a scoping review was deemed appropriate due to the advantages of addressing a wide range of research from an exploratory perspective in order to provide practical implications from a relatively complex body of literature [6].

\section{Eligibility Criteria}

Following the PICOS design of SriHaran, et al. [7], the eligibility criteria included population, intervention, comparator, outcome, and study design. In terms of the population, this study focused on adolescents. In alignment with a contemporary understanding of adolescence, the age range of 10 to 24 years was selected [8]. In terms of intervention, inclusion criteria included empirical studies related to COVID-19 and psychological outcomes among adolescents. Only primary, quantitative studies were considered. As with SriHaran, et al. [7], comparators were not included in the scoping review. As for outcomes, the review focused on biological (physical), psychological, and social risk and preventative factors in the context of psychological wellbeing during the COVID-19 pandemic, including constructs of psychological wellbeing, which also included negative aspects of psychological distress, based on the Depression, Anxiety and Stress Scale-21 (DASS-21) subscales of depression, anxiety, and stress [9]. In terms of study design, manuscripts using quantitative and empirical methods, including those with structural models or clear inferential statistics were selected.

\section{Search Method and Sources}

In order to conduct an expedient and exploratory review of the literature most relevant to psychological wellbeing the PubMed database (offered by the National Library of Medicine, and including literature from MEDLINE as well as other life science journals and online books) was used for the collection of abstracts using the aforementioned keywords. Any data which included the search term "COVID" were included in the analysis, regardless of time. Within the PubMed database, articles were included on the basis of the abstracts containing the key terms of "COVID" and "adolesc*" with the asterisk serving to broaden the search to include terms such as "adolescent" or "adolescence" based on the root, and "AND" being used as a Boolean search operator requiring that both terms be included in the search results. In addition the following terms were also added, in addition to the Boolean search operator of "AND" to yield results specific to the a priori constructs specified by the outcomes aspect of the PICOS model [7], were used in order to collect the initial set of articles by searching the abstracts within PubMed. The specific inclusion terms are as follows: anxiety, biology*, depress*, distress, protect, psych*, risk, social, and stress. As discussed in the results below, in order to exclude non-English and non-empirical studies from the analysis, EndNote9 was used to filter and eliminate irrelevant articles. As such, according to the intervention aspect of the PICOS model [7], the following exclusion terms were used to narrow the results: protocol, review, editorial, and qualitative. The remaining abstracts were further screened (See Figure 1 ), to ensure that all remaining studies were relevant to adolescents, within the age range of 10 to 24 years [8], and specifically mentioned risk and/or protective factors. 


\section{Results}

\section{Search Results}

Based on the search items mentioned above in the eligibility criteria section, a total of 13,212 abstracts were collected. After deleting 6,516 duplicates, a total of 6,696 abstracts were retained for further analysis. As shown in Figure 1, articles were excluded in the case of missing data, not being available in English, using non-empirical methods, and not being clearly related to the adolescent population of interest to this study or not including clear risk or protective factors. At this point, the full text from 281 articles was scrutinized to ensure that the content met the inclusion criteria. Based on a review of these texts, a total of 32 articles were selected for inclusion in this scoping review.

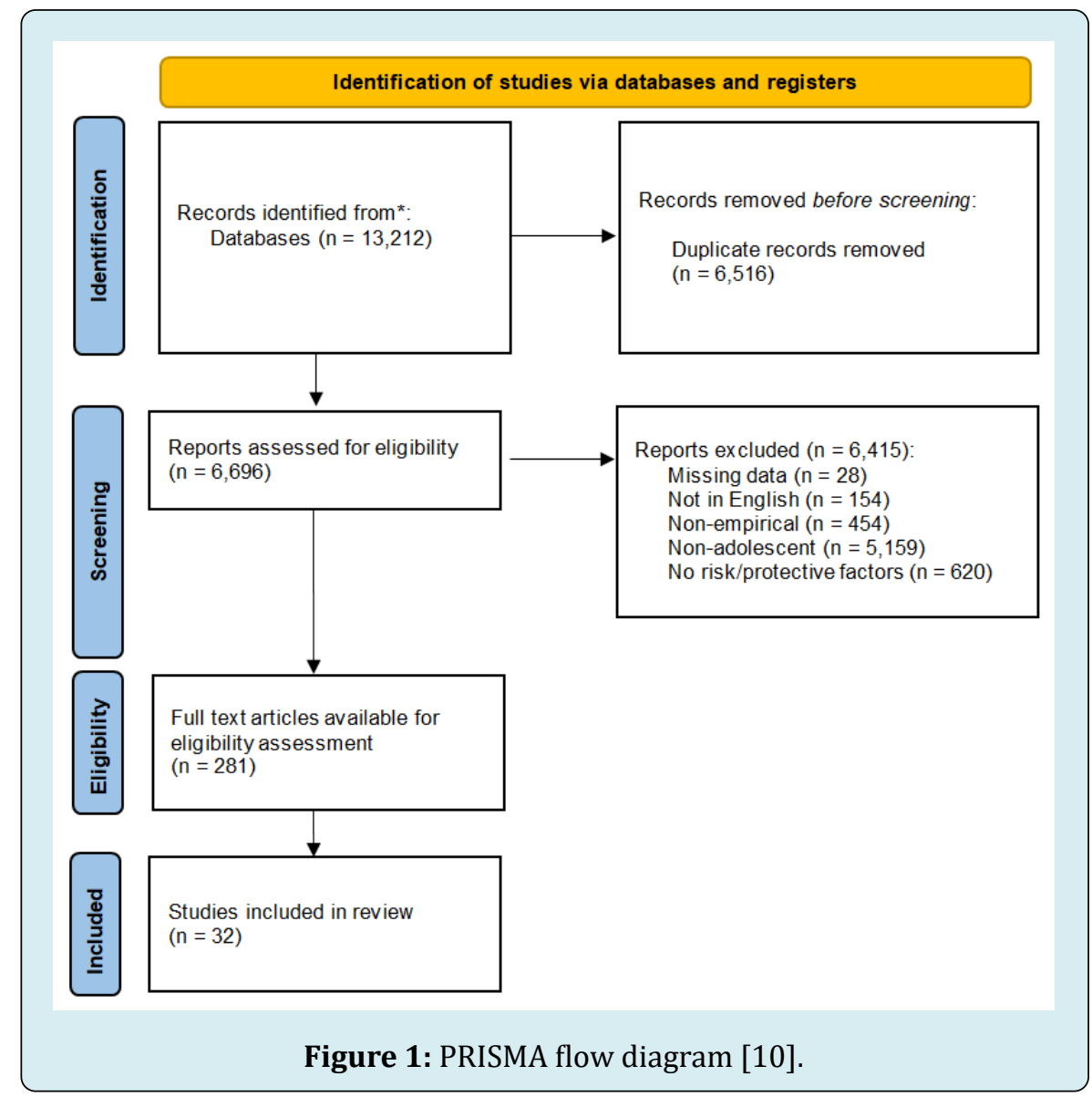

\section{Characteristics of the Selected Studies}

A list of the 32 selected studies is provided in Table 1 , along with the lead author, sample size, study type, risk factors, and protective factors. 11 of the studies conducted longitudinal research, with the other 21 studies conducting cross-sectional research, largely through the use of questionnaires. The scales used by these studies included the Hopkins Symptoms Checklist-25, Multidimensional Perceived Social Support-12 instrument, the Kessler-10 Psychological Distress scale, the COVID-19 Phobia Scale (C19P-S), the Revised Children's Anxiety and Depression Scale (RCADS), a 9-item Patient Health Questionnaire, a 7-item Generalized Anxiety Disorder Scale, a short form of the Childhood Trauma Questionnaire, the Connor-Davidson
Resilience Scale, the Strengths and Difficulties Questionnaire, among others. Self-designed questionnaires were developed for some studies. All studies were empirical in nature, adopting quantitative analysis, and included paired sample t-tests, bivariate analyses, logistic and multinomial logistic regression, mixed model panel regression, cluster analysis, and structural equation modeling.

In general, more risk factors (72) were found than protective factors (41). However, many of these factors overlapped, in that protective factors, when absent or lacking, became risk factors. After categorizing these factors in terms of biological, psychological, and social risk and protective factors, the principle results are reported in the following section. 


\begin{tabular}{|c|c|c|c|c|}
\hline Lead author & $\begin{array}{c}\text { Sample } \\
\text { size }\end{array}$ & Study type & Risk Factors & Protective Factors \\
\hline $\begin{array}{c}\text { Acosta, et al. } \\
{[11]}\end{array}$ & 145 & $\begin{array}{c}\text { Cross-sectional online } \\
\text { survey }\end{array}$ & $\begin{array}{c}\text { female (depression), primary/middle } \\
\text { school (anxiety, depression) }\end{array}$ & $\begin{array}{l}\text { playing sports (anxiety, } \\
\text { depression) }\end{array}$ \\
\hline \multirow{2}{*}{$\begin{array}{l}\text { Akbar, et al. } \\
\quad[12]\end{array}$} & \multirow[b]{2}{*}{250} & \multirow{2}{*}{$\begin{array}{l}\text { Cross-sectional } \\
\text { survey }\end{array}$} & problem-focused coping, & \multirow{2}{*}{$\begin{array}{l}\text { behavioral disengagement, } \\
\text { emotional support (distress) }\end{array}$} \\
\hline & & & $\begin{array}{l}\text { confirmed COVID cases in their area } \\
\text { (distress) }\end{array}$ & \\
\hline $\begin{array}{c}\text { Angelina, et a. } \\
\text { [13] }\end{array}$ & 2,018 & $\begin{array}{l}\text { Cross-sectional } \\
\text { survey }\end{array}$ & $\begin{array}{c}\text { lack of support, argument with } \\
\text { parents, worry, physical or mental } \\
\text { disease (distress) }\end{array}$ & regular diet and sleep (distress) \\
\hline $\begin{array}{l}\text { Bilginer, et al. } \\
{[14]}\end{array}$ & 1,431 & $\begin{array}{c}\text { Cross-sectional online } \\
\text { survey }\end{array}$ & $\begin{array}{c}\text { female, having a healthcare worker } \\
\text { in the residence, physical and mental } \\
\text { disorders, meeting friends in person, } \\
\text { undergoing a CoVID test (anxiety, } \\
\text { depression) }\end{array}$ & \\
\hline Cao, et al. [15] & 11,681 & $\begin{array}{l}\text { Cross-sectional } \\
\text { survey }\end{array}$ & $\begin{array}{l}\text { non-only children, emotional abuse } \\
\text { (anxiety, depression) }\end{array}$ & $\begin{array}{l}\text { resilience, protective parent- } \\
\text { child relationship (anxiety, } \\
\text { depression) }\end{array}$ \\
\hline $\begin{array}{c}\text { Chahal, et al. } \\
{[1]}\end{array}$ & 85 & $\begin{array}{l}\text { Magnetic resonance } \\
\text { imaging }\end{array}$ & low executive control (depression) & \\
\hline $\begin{array}{l}\text { Cohen, et al. } \\
{[16]}\end{array}$ & 24 & $\begin{array}{l}\text { Longitudinal self- } \\
\text { report survey }\end{array}$ & & $\begin{array}{c}\text { talking with friends, prioritizing } \\
\text { sleep (anxiety) }\end{array}$ \\
\hline $\begin{array}{c}\text { Dvorsky, et al. } \\
{[17]}\end{array}$ & 238 & Longitudinal & ADHD (mental health) & $\begin{array}{l}\text { positive coping strategies, } \\
\text { routines (mental health) }\end{array}$ \\
\hline $\begin{array}{l}\text { Esposito, et } \\
\text { al. [2] }\end{array}$ & 2,996 & $\begin{array}{l}\text { Cross-sectional } \\
\text { survey }\end{array}$ & $\begin{array}{l}\text { female (sadness), family member } \\
\text { with COVID (negative feelings) }\end{array}$ & male (negative feelings) \\
\hline $\begin{array}{l}\text { Gatell-Carbó, } \\
\text { et al. [18] }\end{array}$ & 369 & $\begin{array}{l}\text { Longitudinal, } \\
\text { observational }\end{array}$ & worry (mental health problems) & $\begin{array}{c}\text { positive perception of home life } \\
\text { (mental health problems) }\end{array}$ \\
\hline $\begin{array}{l}\text { Gladstone, et } \\
\text { al. [19] }\end{array}$ & 228 & Longitudinal & $\begin{array}{c}\text { negative cognitive style, } \\
\text { dysfunctional attitudes (depression) }\end{array}$ & $\begin{array}{l}\text { resilience, positive relationship } \\
\text { with parents (depression) }\end{array}$ \\
\hline $\begin{array}{c}\text { Iimura, et al. } \\
{[20]}\end{array}$ & 441 & $\begin{array}{c}\text { Cross-sectional } \\
\text { survey }\end{array}$ & & resilience (stress) \\
\hline $\begin{array}{l}\text { Kuhlman, et al. } \\
{[21]}\end{array}$ & 88 & $\begin{array}{l}\text { Cross-sectional } \\
\text { survey } \\
\end{array}$ & $\begin{array}{l}\text { COVID-19 experiences of events and } \\
\text { concerns (depression, aggression) }\end{array}$ & $\begin{array}{c}\text { cognitive reappraisal, humor } \\
\text { (resilience, mental health) }\end{array}$ \\
\hline Liao, et al. [22] & 2,496 & Longitudinal study & shortened sleep (depression) & $\begin{array}{l}\text { exercise duration (depression } \\
\text { mediated by sleep) }\end{array}$ \\
\hline Liu, et al. [23] & 1,594 & $\begin{array}{c}\text { Cross-sectional multi- } \\
\text { source assessment }\end{array}$ & $\begin{array}{l}\text { lack of routine, child-parent conflict } \\
\text { (loneliness, depression) }\end{array}$ & \\
\hline Liu, et al. [24] & 617 & $\begin{array}{c}\text { Cross-sectional online } \\
\text { survey }\end{array}$ & perceived stress (depression) & $\begin{array}{l}\text { character strengths } \\
\text { (depression) }\end{array}$ \\
\hline $\begin{array}{l}\text { Magson, et al. } \\
{[25]}\end{array}$ & 248 & Longitudinal study & $\begin{array}{l}\text { COVID-19 worries, conflict with } \\
\text { parents (mental health) }\end{array}$ & $\begin{array}{l}\text { compliance with lockdown } \\
\text { orders, social connectedness } \\
\text { (mental health) }\end{array}$ \\
\hline $\begin{array}{l}\text { McKune, et al. } \\
{[26]}\end{array}$ & 280 & $\begin{array}{l}\text { Cross-sectional } \\
\text { survey }\end{array}$ & $\begin{array}{c}\text { loss of income (anxiety), female } \\
\text { (depression, anxiety), over-protective } \\
\text { parenting (depression), younger } \\
\text { (anxiety) }\end{array}$ & \\
\hline
\end{tabular}




\begin{tabular}{|c|c|c|c|c|}
\hline $\begin{array}{l}\text { Oliva, et al. } \\
\quad[27]\end{array}$ & 9,688 & $\begin{array}{l}\text { Cross-sectional } \\
\text { anonymous online } \\
\text { survey }\end{array}$ & $\begin{array}{c}\text { male, autistic, COVID in the area, } \\
\text { parental job loss, screen time (mental } \\
\text { health disorders) }\end{array}$ & $\begin{array}{c}\text { having siblings, physical activity } \\
\text { home-schooling, reading, } \\
\text { talking with others (mental } \\
\text { health disorders) }\end{array}$ \\
\hline $\begin{array}{l}\text { Paschke, et al. } \\
{[28]}\end{array}$ & 1,221 & $\begin{array}{l}\text { Longitudinal } \\
\text { observational online } \\
\text { survey } \\
\end{array}$ & $\begin{array}{c}\text { female parent, financial worries, } \\
\text { negative emotional regulation } \\
\text { (stress) }\end{array}$ & \\
\hline Qin, et al. [29] & 254 & Longitudinal study & no siblings, stem families (anxiety) & exercising (mental health) \\
\hline Qin, et al. [30] & $11,99,320$ & $\begin{array}{l}\text { Cross-sectional } \\
\text { survey }\end{array}$ & $\begin{array}{l}\text { high school students, non-mask } \\
\text { wearing, low levels of exercise } \\
\text { (distress) }\end{array}$ & \\
\hline $\begin{array}{c}\text { Ravens- } \\
\text { Sieberer, et al. } \\
{[31]} \\
\end{array}$ & 1,923 & $\begin{array}{c}\text { Two-wave } \\
\text { longitudinal study }\end{array}$ & $\begin{array}{l}\text { social disadvantage, mentally } \\
\text { burdened parents (mental health) }\end{array}$ & $\begin{array}{l}\text { female, older, positive family } \\
\text { climate, social support (mental } \\
\text { health) }\end{array}$ \\
\hline Raw, et al. [32] & 2,988 & Longitudinal study & $\begin{array}{c}\text { distressed parent, special educational } \\
\text { needs, neurodevelopmental } \\
\text { disorders, younger (distress) }\end{array}$ & \\
\hline Ren, et al. [33] & 1,487 & $\begin{array}{l}\text { Cross-sectional } \\
\text { survey }\end{array}$ & $\begin{array}{l}\text { community infection, older } \\
\text { adolescents (mental health) }\end{array}$ & $\begin{array}{l}\text { physical activity, daily life } \\
\text { routines (mental health) }\end{array}$ \\
\hline Ren, et al. [34] & 1,771 & Cross-sectional study & $\begin{array}{l}\text { poor sleep quality, female, infected } \\
\text { family members, low subjective } \\
\text { school or social status (mood } \\
\text { disorders) }\end{array}$ & \\
\hline $\begin{array}{l}\text { Rosen, et al. } \\
\quad[35]\end{array}$ & 224 & Longitudinal study & $\begin{array}{l}\text { exposure to pandemic-related } \\
\text { stressors (psychopathological } \\
\text { symptoms) }\end{array}$ & $\begin{array}{l}\text { structured routine, less passive } \\
\text { screen time, lower exposure } \\
\text { to news, time in nature, better } \\
\text { sleep (psychopathological } \\
\text { symptoms) }\end{array}$ \\
\hline $\begin{array}{l}\text { Wang, et al. } \\
\text { [36] }\end{array}$ & 12,186 & Cross-sectional study & \begin{tabular}{|c} 
parent-child conflict, sleep problems, \\
video game play, eating disorders \\
(psychological problems)
\end{tabular} & $\begin{array}{c}\text { physical activity (psychological } \\
\text { problems) }\end{array}$ \\
\hline $\begin{array}{l}\text { Wang, et al. } \\
\text { [37] }\end{array}$ & 11,072 & Cross-sectional study & $\begin{array}{c}\text { attending reopened schools, parent- } \\
\text { child conflict, sedentary lifestyle, } \\
\text { sleep problems (psychosocial and } \\
\text { behavioral problems) }\end{array}$ & \\
\hline Wu, et al. [38] & 4,807 & Cross-sectional study & poor family functioning (stress) & \\
\hline $\begin{array}{l}\text { Zhang, et al. } \\
\text { [39] }\end{array}$ & 1,025 & $\begin{array}{l}\text { Cross-sectional online } \\
\text { study }\end{array}$ & $\begin{array}{c}\text { negative coping (depression, anxiety, } \\
\text { stress) }\end{array}$ & $\begin{array}{l}\text { resilience, positive coping } \\
\text { (depression, anxiety, stress) }\end{array}$ \\
\hline $\begin{array}{l}\text { Zhou, et al. } \\
\quad[40]\end{array}$ & 8,079 & Cross-sectional study & $\begin{array}{l}\text { female, higher grade level } \\
\text { (depression, anxiety) }\end{array}$ & \\
\hline
\end{tabular}

Table 1: Summary of the selected studies.

\section{Biopsychosocial Risk Factors}

Biological Risk Factors: Mixed results were found in terms of sex, with one study suggesting that males at greater risk of negative feelings [27], but with a total of seven studies identifying females as being at greater risk of several mental health issues $[2,11,14,26,34,40]$. Findings related to age are likewise mixed, with some evidence for higher risk of anxiety and depression among primary and middle school students [11], with other studies reporting greater risk, over time, for older adolescents $[33,40]$ and high school students [30]. Pre-existing physical diseases are a risk factor for distress, anxiety, and depression [13,14], including ADHD [17], autism [27], neurodevelopmental disorder [32], and 
eating disorders [36]. Sleep is another commonly reported risk factor $[22,34,36,37]$ in terms of depression, mood and psychological disorders, and psychosocial and behavioral problems). Likewise, lack of exercise [30], and a sedentary lifestyle [37], are considered as risk factors for distress and psychosocial and behavioral issues.

\section{Psychological Risk Factors}

While coping strategies are generally considered protective factors, problem-focused [12], or negative coping strategies [39], may serve as risk factors for mental distress. In a related manner, cognitive aspects, such as low executive control [1], negative emotional regulation [28], negative cognitive style [19], and dysfunctional attitudes [19], were reported as risk factors in the literature. Worry is a risk factor for psychological distress $[13,18,25]$, including financial worries [28]. Just as physical disease served as a risk factor for psychological distress, mental disease $[13,14]$ and special educational needs [32] are psychological risk factors. Emotional abuse [15], is associated with anxiety and depression.

\section{Social Risk Factors}

Several social risk factors have been reported, many of which relate to being exposed to COVID-19, such as having a diagnosed family member [2,34], having cases in their community $[12,27,33]$, or experiencing events or concerns related to COVID-19 [21], or related stressors [35], as well as taking the risk of meeting friends in person [14], and undergoing COVID-19 testing [14]. Given the risk of COVID-19 in the community, the role of social support becomes important, with lack of support resulting in distress [13], social disadvantages [31], loss of income or parents' employment [26,27], and low subjective school or social status [34], serving as risk factors for mental health and mood disorders. The role of the family in offering positive social support is, likewise, a tangible need for adolescents. As such, risk factors for psychological stress include arguments or conflict with parents $[23,25,36,37]$, poor family functioning [38], over-protective parenting [26], and having mentally burdened or distressed parents [31,32]. One important factor related to family life is that of routine, with adolescents lacking routine being at greater risk for anxiety or depression [23]. Interestingly, other family and community factors, such as having a healthcare worker in the residence [14], siblings $[15,29]$, and belonging to stem families [29], also serve as risk factors for anxiety and depression. Social behaviors such as attending reopened schools, as compared to being homeschooled [37], having increased screen time or video game play [27], and not wearing masks [36], as per mandates are additional risk factors for adolescents.

\section{Biopsychosocial Protective Factors}

Biological Protective Factors: As with risk factors, sex as a protective factor was not reported consistently in the literature, with studies associating the male sex with risk of negative feelings [2], or the female sex with risk of mental health [31]. While one study reported that older adolescents were less at risk of mental health issues (age as a protective factor) [31], as with the findings from biological risk factors, it is difficult to make clear interpretations. However, in terms of biological well-being factors related to healthy behaviors, there is ample evidence of the importance of sports and physical activity $[11,22,29,33,36]$, time spent in natural environments [35], regular diet [13], and sufficient sleep $[1316,22,25]$, as protective factors in terms of adolescents' psychological wellbeing during COVID-19.

\section{Psychological Protective Factors}

In line with several recent studies emphasizing resilience, the role of resilience as a protective factor was a common theme among the articles selected for this scoping review $[15,19,20,21,39]$, as with related constructs such as character strengths [24]. Additionally, positive coping was found to be a protective factor overall $[17,39]$, as well as in terms of cognitive reappraisal and humor [21], behavioral disengagement [12], and talking with friends or others $[16,27]$. Other behaviors that were reported as protective factors include reducing passive screen time [35], reading [27], avoiding exposure to news [35], and compliance with lockdown orders [25].

\section{Social Protective Factors}

In terms of social protective factors, emotional and social support, family relationships, and routines are key to mitigating the effects of COVID-19 on adolescent psychological well-being. Emotional support [12], and social support and connectedness [31], were reported as protective factors. In terms of adolescents' home life, protective parent-child relationships [15], positive perceptions of home life [18], positive relationships with parents [19], positive family climate [31], and home-schooling [27], were reported as protective factors. Daily life routines $[17,33]$ and structured routine [35], were also predictive of more positive psychological outcomes for adolescents during the COVID-19 pandemic.

\section{Discussion}

\section{Summary of Findings}

From a pool of 281 potential articles, 32 studies with quantitative research designs related to adolescent 
psychological wellbeing, which reported risk and protective factors during the COVID-19 pandemic, were evaluated in this scoping review. Overall, in terms of physical factors influencing adolescent psychological wellbeing, it appears that females are more at risk as compared to males, although the results are somewhat mixed. Pre-existing physical and mental conditions also serve as clear risk factors during the pandemic, as individuals with both physical and mental disorders or either physical or mental disorders are disproportionately impacted by the COVID-19 pandemic [41]. Awareness of this risk may also be known to individuals with physical or mental conditions, which may compound the psychological impact through increased fear of COVID-19 [42]. In terms of biological well-being factors related to healthy behaviors, there is evidence of the importance of sports and physical activity, diet, and sufficient sleep. In terms of psychological factors, negative coping strategies, such as those that are problem-focused, and other cognitive factors, including low executive control, emotional regulation and dysfunctional or negative attitudes can increase the risk of psychological distress during the pandemic. Worry and mental disease were also clearly linked to risk of psychological distress. Alternatively, resilience, positive coping strategies, and other steps to avoid COVID-19 news and reduce screen time were protective factors for adolescents. Finally, in terms of social factors, having COVID-19 cases in the community or family were strong risk factors for psychological distress. Lack of social support, including negative family functioning or conflicts in the home, was also predictive of psychological distress among adolescents during the pandemic. On the other hand, emotional and social support, family relationships, and routines were key to mitigating the effects of COVID-19 on adolescent psychological well-being.

\section{Limitations}

The scoping review was conducted by the author and without assistance by other raters. This limits the reliability and validity of the protocol. While the PRISMA-ScR Checklist (see the Appendix) was followed as closely as possible, data charting was not clearly discussed.

Moreover, while each of the retained articles was read carefully, there were no clearly stated standards of critical appraisal adopted, such as the rigor of the statistical analysis. Likewise, the use of a single database (PubMed) limited the number of potential quantitative studies available for analysis. As the content of a single database introduces the potential for bias, further research addressing risk and protective factors in the context of COVID-19 should include other databases, such as PsycINFO and ERIC. As the resulting articles included in this analysis may not be completely representative, and potentially skewed towards more biological/physical and psychological-oriented journals, future studies can index databases and journals that may include more social risk and protective factors through a more thorough and expansive search of relevant literature.

\section{Conclusion}

Based on the scoping review presented in this paper, the following implications are suggested for researchers and practitioners:

> Given the fact that homeschooling and family relationships served as protective factors for adolescents' psychological wellbeing while attending reopened schools, and meeting friends in person served as risk factors, schools and policy-makers should take great care in making decisions regarding opening of schools.

$>$ Basic health behaviors, including proper sleep, diet, and physical activity should be encouraged during lockdown or similar restrictive measures. If online teaching is implemented, the integration of activities and lessons targeting these key behaviors could improve psychological outcomes for adolescents.

$>$ In allocating resources during the pandemic, particularly in terms of psychological or psychiatric care, it is important to consider the relatively stronger impact of the pandemic on individuals with pre-existing physical and mental conditions.

$>$ The role of the family and social support (or lack thereof) as both protective and risk factors should inform the development of family education in order to develop stronger relationships between adolescents and their parents, providing both educational and financial resources for parents at risk of family dysfunction, or who have reported issues such as conflict or abuse. Specific strategies can include reducing screen time, establishing structures and routines, and promoting parent-child physical activity.

$>$ Given the importance of resilience and coping in mitigating the effects of the pandemic on adolescents' psychological wellbeing, explicit instruction on appropriate coping strategies and the provisions of teacher support should be provided, in order to reduce negative emotions and develop adolescents' resilience [43].

\section{Funding}

The author received no financial support for this research.

\section{Supplemental Material}

A table including the reporting items outlined by the Preferred Reporting Items for Systematic Reviews and MetaAnalyses (PRISMA) checklist provided for scoping reviews 
[5] is provided as an appendix.

\section{References}

1. Chahal R, Kirshenbaum JS, Miller JG, Ho TC, Gotlib IH (2021) Higher executive control network coherence buffers against puberty-related increases in internalizing symptoms during the COVID-19 pandemic. Biol Psychiatry Cogn Neurosci Neuroimaging 6(1): 79-88.

2. Esposito S, Giannitto N, Squarcia A, Neglia C, Argentiero A, et al. (2020) Development of psychological problems among adolescents during school closures because of the COVID-19 lockdown phase in Italy: A cross-sectional survey. Front Pediatr 8: 628072.

3. Yi J, Chen IH, Lin CY, Li CC, Liao XL, et al. (2021) The effect of primary and middle school teachers' problematic internet use and fear of COVID-19 on psychological need thwarting of online teaching and psychological distress. Healthcare (Basel) 9(9): 1199.

4. Peters MDJ, Godfrey C, McInerney P, Munn Z, Tricco AC, et al. (2020) Chapter 11: Scoping reviews. In: Aromataris E, Munn Z, et al. (Eds.), JBI reviewer's manual, Joanna Briggs Institute, Adelaide. Australia.

5. Tricco AC, Lillie E, Zarin W, O Brien KK, Colquhoun $\mathrm{H}$, et al. (2018) PRISMA Extension for Scoping Reviews (PRISMAScR): Checklist and Explanation. Ann Intern Med 169(7): 467-473.

6. Daniel M, Rencic J, Durning SJ, Holmboe E, Santen SA, et al. (2019) Clinical reasoning assessment methods: a scoping review and practical guidance. Acad Med 94(6): 902-912.

7. Sriharan A, Ratnapalan S, Tricco A, Lupea D, Ayala AP, et al. (2020) Occupational stress, burnout and depression in women in healthcare during COVID-19 pandemic: A rapid scoping review. Front Glob Womens Health 1: 596690.

8. Sawyer SM, Azzopardi PS, Wickremarathne D, Patton GC (2018) The age of adolescence. The Lancet Child Adolesc Health 2(3): 223-228.

9. Lovibond SH, Lovibond PF (1995) Manual for the Depression Anxiety Stress Scales. Psychology Foundation of Australia, Sydney, Australia.

10. Page MJ, McKenzie JE, Bossuyt PM, Boutron I, Hoffmann TC, et al. (2021) The PRISMA 2020 statement: An updated guideline for reporting systematic reviews. BMJ 372: $\mathrm{n}(71)$.
11. Acosta D, Fujii Y, Beaulieu DJ, Jacobs KD, Maurelli AT, et al. (2021) Psychosocial health of K-12 students engaged in emergency remote education and in-person schooling: A cross-sectional study. Int J Environ Res Public Health 18(16): 8564.

12. Akbar Z, Aisyawati MS (2021) Coping strategy, social support, and psychological distress among university students in Jakarta, Indonesia during the COVID-19 pandemic. Front Psychol 12: 694122.

13. Angelina S, Kurniawan A, Agung FH, Halim DA, Wijovi F, et al. (2021) Adolescents' mental health status and influential factors amid the Coronavirus Disease pandemic. Clin Epidemiol Glob Health 12: 100903.

14. Bilginer C, Yildirim S, Yilmaz BC, Beyhun E, Karadeniz S (2021) Changes in adolescent mental health during the covid pandemic. Minerva Pediatr (Torino).

15. Cao Y, Huang L, Si T, Wang NQ, Qu M, et al. (2021) The role of only-child status in the psychological impact of COVID-19 on mental health of Chinese adolescents. J Affect Disord 282: 316-321.

16. Cohen ZP, Cosgrove KT, DeVille DC, Akeman E, Singh MK, et al. (2021) The impact of COVID-19 on adolescent mental health: Preliminary findings from a longitudinal sample of healthy and at-risk adolescents. Front Pediatr 9: 622608 .

17. Dvorsky MR, Breaux R, Cusick CN, Fredrick JW, Green C, et al. (2021) Coping with COVID-19: Longitudinal impact of the pandemic on adjustment and links with coping for adolescents with and without ADHD. Res Child Adolesc Psychopathol pp: 1-15.

18. Carbó AG, Bloch EA, Martínez JVB, Porcuna TP, Carceller ME, et al. (2021) State of child and adolescent mental health during the first wave of the COVID-19 pandemic and at the beginning of the 2020-2021 school year. An Pediatr (Engl Ed) 95(5): 354-363.

19. Gladstone TRG, Schwartz JAJ, Pössel P, Richer AM, Buchholz KR, et al. (2021) Depressive symptoms among adolescents: Testing vulnerability-stress and protective models in the context of COVID-19. Child Psychiatry Hum Dev pp: 1-11.

20. Iimura S (2022) Sensory-processing sensitivity and COVID-19 stress in a young population: The mediating role of resilience. Pers Individ Dif 184: 111183.

21. Kuhlman KR, Straka K, Mousavi Z, Tran ML, Rodgers E (2021) Predictors of adolescent resilience during the COVID-19 pandemic: Cognitive reappraisal and humor. J 
Adolesc Health 69(5): 729-736.

22. Liao S, Luo B, Liu H, Zhao L, Shi W, et al. (2021) Bilateral associations between sleep duration and depressive symptoms among Chinese adolescents before and during the COVID-19 pandemic. Sleep Med 84: 289-293.

23. Liu J, Zhou T, Yuan M, Ren H, Bian X, et al. (2021) Daily routines, parent-child conflict, and psychological maladjustment among Chinese children and adolescents during the COVID-19 pandemic. J Fam Psychol.

24. Liu Q Wang Z (2021) Perceived stress of the COVID-19 pandemic and adolescents' depression symptoms: The moderating role of character strengths. Pers Individ Dif 182: 111062.

25. Magson NR, Freeman JYA, Rapee RM, Richardson CE, Oar EL, et al. (2021) Risk and protective factors for prospective changes in adolescent mental health during the COVID-19 pandemic. J Youth Adolesc 50(1): 44-57.

26. McKune SL, Acosta D, Diaz N, Brittain K, Beaulieu DJ, et al. (2021) Psychosocial health of school-aged children during the initial COVID-19 safer-at-home school mandates in Florida: a cross-sectional study. BMC Public Health 21: 603.

27. Oliva S, Russo G, Gili R, Russo L, Mauro AD, et al. (2021) Risks and protective factors associated with mental health symptoms during COVID-19 home confinement in Italian children and adolescents: The \#Understandingkids study. Front Pediatr 9: 664702.

28. Paschke K, Arnaud N, Austermann MI, Thomasius $R$ (2021) Risk factors for prospective increase in psychological stress during COVID-19 lockdown in a representative sample of adolescents and their parents. BJPsych Open 7(3): e94.

29. Qin J, Ding Y, Gao J, Wu Y, Lv H, et al. (2021) Effects of COVID-19 on mental health and anxiety of adolescents Aged 13-16 years: A comparative analysis of longitudinal data from China. Front Psychiatry 12: 695556.

30. Qin Z, Shi L, Xue Y, Lin H, Zhang J, et al. (2021) Prevalence and risk factors associated with self-reported psychological distress among children and adolescents during the COVID-19 pandemic in China. JAMA Netw Open 4(1): e2035487.

31. Sieberer UR, Kaman A, Erhart M, Otto C, Devine J, et al. (2021) Quality of life and mental health in children and adolescents during the first year of the COVID-19 pandemic: results of a two-wave nationwide populationbased study. Eur Child Adolesc Psychiatry pp: 1-14.
32. Raw JAL, Waite P, Pearcey S, Shum A, Patalay P, et al. (2021) Examining changes in parent-reported child and adolescent mental health throughout the UK's first COVID-19 national lockdown. J Child Psychol Psychiatry 62(12): 1391-1401.

33. Ren H, He X, Bian X, Shang X, Liu J (2021) The protective roles of exercise and maintenance of daily living routines for Chinese adolescents during the COVID-19 quarantine period. J Adolesc Health 68(1): 35-42.

34. Ren Z, Xin Y, Wang Z, Liu D, Ho RCM, et al. (2021) What factors are most closely associated with mood disorders in adolescents during the COVID-19 pandemic? A crosssectional study based on 1,771 adolescents in Shandong Province, China. Front Psychiatry 12: 728278.

35. Rosen ML, Rodman AM, Kasparek SW, Mayes M, Freeman MM, et al. (2021) Promoting youth mental health during the COVID-19 pandemic: A longitudinal study. PLoS One 16(8): e0255294.

36. Wang L, Chen L, Jia F, Shi X, Zhang Y, et al. (2021) Risk factors and prediction nomogram model for psychosocial and behavioural problems among children and adolescents during the COVID-19 pandemic: A national multicentre study: Risk factors of childhood psychosocial problems. J Affect Disord 294: 128-136.

37. Wang L, Zhang Y, Chen L, Wang J, Jia F, et al. (2021) Psychosocial and behavioral problems of children and adolescents in the early stage of reopening schools after the COVID-19 pandemic: a national cross-sectional study in China. Transl Psychiatry 11(1): 342.

38. Wu Z, Zou Z, Wang F, Xiang Z, Zhu M, et al. (2021) Family functioning as a moderator in the relation between perceived stress and psychotic-like experiences among adolescents during COVID-19. Compr Psychiatry 111: 152274 .

39. Zhang C, Ye M, Fu Y, Yang M, Luo F, et al. (2020) The psychological impact of the COVID-19 pandemic on teenagers in China. J Adolesc Health 67(6): 747-755.

40. Zhou SJ, Zhang LG, Wang LL, Guo ZC, Wang JQ et al. (2020) Prevalence and socio-demographic correlates of psychological health problems in Chinese adolescents during the outbreak of COVID-19. Eur Child Adolesc Psychiatry 29(6): 749-758.

41. Alonzi S, La Torre A, Silverstein MW (2020) The psychological impact of preexisting mental and physical health conditions during the COVID-19 pandemic. Psychol Trauma 12(S1): S236-S238. 
42. Buneviciene I, Bunevicius R, Bagdonas S, Bunevicius A (2021) The impact of pre-existing conditions and perceived health status on mental health during the COVID-19 pandemic. J Public Health (Oxf) fdab248.
43. Guo J, Liu L, Zhao B, Wang D (2020) Teacher support and mental well-being in Chinese adolescents: the mediating role of negative emotions and resilience. Front Psychol 10: 3081 . 